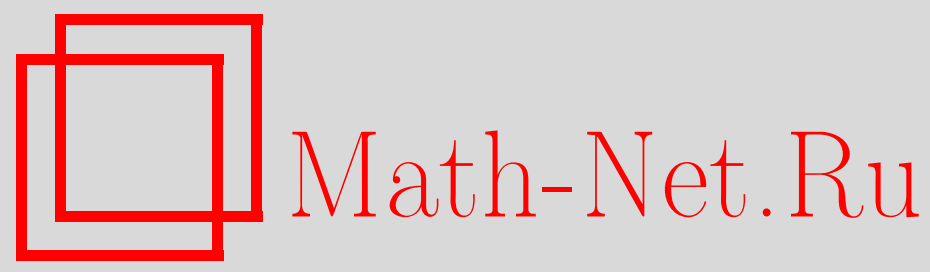

В. А. Краснов, О фундаментальных классах гомологий вещественного алгебраического многообразия, Матем. заметки, 1999, том 66, выпуск 2, 216-219

DOI: https://doi.org/10.4213/mzm1159

Использование Общероссийского математического портала Math-Net.Ru подразумевает, что вы прочитали и согласны с пользовательским соглашением http://www.mathnet.ru/rus/agreement

Параметры загрузки:

IP : 34.229 .108 .108

26 апреля 2023 г., 12:05:50

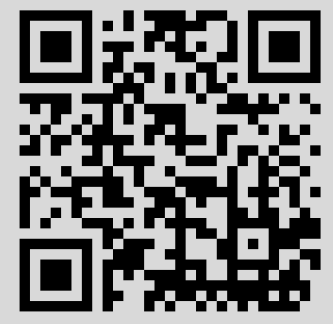




\section{О ФУНДАМЕНТАЛЬНЫХ КЛАССАХ ГОМОЛОГИЙ ВЕЩЕСТВЕННОГО АЛГЕБРАИЧЕСКОГО МНОГООБРАЗИЯ}

В. А. Краснов

Доказано, что между классами гомологий, определенных вещественными точками специалного вещественного алгебраического многообразия, может существовать только одно соотношение. Это соотношение равно сумме всех классов гомологий.

Библиография: 4 названия.

Введение. Далее $X$ - неособое вешественное проективное многообразие. Пусть $X_{1}, \ldots, X_{s}$ - компоненты связности множества вещественных точек $X(\mathbb{R})$. Они определяют классы гомологий $\left[X_{1}\right], \ldots,\left[X_{s}\right] \in H_{n}\left(X(\mathbb{C}), \mathbb{F}_{2}\right)$, где $n=\operatorname{dim} X$. Возникает вопрос, какие соотношения существуют между этими классами гомологий? Для кривой может быть только одно соотношение, а именно: $\left[X_{1}\right]+\cdots+\left[X_{s}\right]=0$, т.е. $[X(\mathbb{R})]=0$. В работе [1] показано, что для поверхности с $H_{1}\left(X(\mathbb{C}), \mathbb{F}_{2}\right)=0$ также может быть только одно соотношение $[X(\mathbb{R})]=0$. С помощю эквивариантных когомологий аналогичное утверждение было доказано в [2] для полных пересечений произвольной размерности. В данной заметке мы обобщаем сформулированное вьше утверждение на класс "специальных" многообразий. Мы называем многообразие $X$ сnециальным, если отображение цикла

$$
\operatorname{cl}_{\mathbb{C}}: A^{<n / 2}(X) \rightarrow H^{<n}\left(X(\mathbb{C}), \mathbb{F}_{2}\right)
$$

эпиморфно, где $A^{<n / 2}(X)$ - прямая сумма групп Чжоу $A^{q}(X)$ при $q<n / 2$, а $H^{<n}\left(X(\mathbb{C}), \mathbb{F}_{2}\right)$ - прямая сумма групш когомологий $H^{p}\left(X(\mathbb{C}), \mathbb{F}_{2}\right)$ при $p<n$.

ТеоремА. Если $X$ - специальное многообразие, то среди классов гомологий $\left[X_{1}\right], \ldots,\left[X_{s}\right] \in H_{n}\left(X(\mathbb{C}), \mathbb{F}_{2}\right)$ мохет быть только одно соотношение $[X(\mathbb{R})]=0$.

Заметим, что из этой теоремы вытекают сформулированные ранее утверждения о кривой, поверхности и полном пересечении. Доказательство теоремы, изложенное в данной заметке, использует эквивариантные когомологии и отображения цикла. Именно, применение отображений цикла позволило обобщить результат в [2].

1. Эквивариантные когомологии и отображения цикла. Рассмотрим кольцо эквивариантных когомологий

$$
H^{*}\left(X(\mathbb{C}) ; G, \mathbb{F}_{2}\right)=\bigoplus_{q \geqslant 0} H^{q}\left(X(\mathbb{C}) ; G, \mathbb{F}_{2}\right),
$$


где $G=G(\mathbb{C} / \mathbb{R})$ - групша Галуа. Оно является алгеброй над кольцом многочленов

$$
H^{*}\left(\mathrm{pt} ; G, \mathbb{F}_{2}\right)=H^{*}\left(G, \mathbb{F}_{2}\right)=\mathbb{F}_{2}[t]
$$

где $\mathrm{pt}$ - одноточечное пространство, $t$ - образующая групшы $H^{1}\left(G, \mathbb{F}_{2}\right)$. Заметим, что структурньй гомоморфизм

$$
H^{*}\left(\mathrm{pt} ; G, \mathbb{F}_{2}\right) \rightarrow H^{*}\left(X(\mathbb{C}) ; G, \mathbb{F}_{2}\right)
$$

индуцирован отображением $X(\mathbb{C}) \rightarrow$ pt. Рассмотрим три гомоморфизма:

$$
\begin{aligned}
& \alpha^{*}: H^{*}\left(X(\mathbb{C}) ; G, \mathbb{F}_{2}\right) \rightarrow H^{*}\left(X(\mathbb{C}), \mathbb{F}_{2}\right), \\
& \beta^{*}: H^{*}\left(X(\mathbb{C}) ; G, \mathbb{F}_{2}\right) \rightarrow H^{*}\left(X(\mathbb{R}) ; G, \mathbb{F}_{2}\right), \\
& \mathrm{cl}^{*}: A^{*}(X) \rightarrow H^{*}\left(X(\mathbb{C}) ; G, \mathbb{F}_{2}\right),
\end{aligned}
$$

где $\alpha^{*}$ - гомоморфизм забьвания вещественной структуры, $\beta^{*}$ - гомоморфизм ограничения, $A^{*}(X)$ - кольцо Чжоу и $\mathrm{cl}^{*}$ - отображение цикла. Гомоморфизмы $\alpha^{*}, \mathrm{cl}^{*}$ являются гомоморфизмами колец, а $\beta^{*}$ есть гомоморфизм $\mathbb{F}_{2}[t]$-алгебр. Заметим, что

$$
H^{q}\left(X(\mathbb{R}) ; G, \mathbb{F}_{2}\right)=\bigoplus_{k=0}^{q} H^{k}\left(X(\mathbb{R}), \mathbb{F}_{2}\right)
$$

а гомоморфизм умножения на $t$

$$
H^{q}\left(X(\mathbb{R}) ; G, \mathbb{F}_{2}\right) \rightarrow H^{q+1}\left(X(\mathbb{R}) ; G, \mathbb{F}_{2}\right)
$$

является вложением прямой суммы в прямую сумму, т.е. $t \cup a=a$, где $a \in H^{k}\left(X(\mathbb{R}), \mathbb{F}_{2}\right)$. Пусть $n=\operatorname{dim} X$. Обозначим через $K^{n}\left(X(\mathbb{C}) ; G, \mathbb{F}_{2}\right)$ ядро гомоморфизма

$$
\alpha^{n}: H^{n}\left(X(\mathbb{C}) ; G, \mathbb{F}_{2}\right) \rightarrow H^{n}\left(X(\mathbb{C}), \mathbb{F}_{2}\right)
$$

Тогда выполняется равенство

$$
K^{n}\left(X(\mathbb{C}) ; G, \mathbb{F}_{2}\right)=t \cup H^{n-1}\left(X(\mathbb{C}) ; G, \mathbb{F}_{2}\right)
$$

Это равенство вытекает из спектральной последовательности

$$
\mathrm{II}_{2}^{p, q}=H^{p}\left(G, H^{q}\left(X(\mathbb{C}), \mathbb{F}_{2}\right)\right) \Longrightarrow H^{p+q}\left(X(\mathbb{C}) ; G, \mathbb{F}_{2}\right)
$$

так как гомоморфизм $\alpha^{n}$ является ее краевым гомоморфизмом. Пусть $2 q<n$. Тогда $t^{n-2 q} \cup \mathrm{cl}^{q}\left(A^{q}(X)\right)$ является подгрупой $K^{n}\left(X(\mathbb{C}) ; G, \mathbb{F}_{2}\right)$. Этот факт вытекает из равенства $(1)$. Обозначим через $K_{\mathrm{alg}}^{n}\left(X(\mathbb{C}) ; G, \mathbb{F}_{2}\right)$ сумму подгрупп $t^{n-2 q} \cup \mathrm{cl}^{q}\left(A^{q}(X)\right)$ при $2 q<n$. Если $X-$ специальное многообразие, то выполняется равенство

$$
K_{\mathrm{alg}}^{n}\left(X(\mathbb{C}) ; G, \mathbb{F}_{2}\right)=K^{n}\left(X(\mathbb{C}) ; G, \mathbb{F}_{2}\right)
$$


Это равенство вытекает из определения специального многообразия и спектральной последовательности (2). В работе [3] была вычислена композиция гомоморфизмов

$$
\beta^{2 q} \circ \mathrm{cl}^{q}: A^{q}(X) \rightarrow H^{2 q}\left(X(\mathbb{R}) ; G, \mathbb{F}_{2}\right)=\bigoplus_{k=0}^{2 q} H^{k}\left(X(\mathbb{R}), \mathbb{F}_{2}\right),
$$

а именно: если $\mathrm{cl}_{\mathbb{R}}^{q}: A^{q}(X) \rightarrow H^{q}\left(X(\mathbb{R}), \mathbb{F}_{2}\right)$ - вещественное отображение цикла, то

$$
\beta^{2 q} \circ \mathrm{cl}^{q}=\mathrm{Sq} \circ \mathrm{cl}_{\mathbb{R}}^{q}
$$

где $\mathrm{Sq}$ - полньй квадрат Стинрода. Через

$$
\beta^{n, 0}: H^{n}\left(X(\mathbb{C}) ; G, \mathbb{F}_{2}\right) \rightarrow H^{0}\left(X(\mathbb{R}), \mathbb{F}_{2}\right)
$$

обозначим композицию гомоморфизма $\beta^{n}$ с проекцией

$$
\bigoplus_{k=0}^{n} H^{k}\left(X(\mathbb{R}), \mathbb{F}_{2}\right) \rightarrow H^{0}\left(X(\mathbb{R}), \mathbb{F}_{2}\right)
$$

Тогда из (4) вытекает равенство

$$
\beta^{n, 0}\left(K_{\mathrm{alg}}^{n}\left(X(\mathbb{C}) ; G, \mathbb{F}_{2}\right)\right)=\mathbb{F}_{2} \cdot x^{0}
$$

где $x^{0} \in H^{0}\left(X(\mathbb{R}), \mathbb{F}_{2}\right)$ - класс когомологий, которьй на каждом одноточечном цикле принимает значение 1. Для специального многообразия из $(3),(5)$ получаем равенство

$$
\beta^{n, 0}\left(K^{n}\left(X(\mathbb{C}) ; G, \mathbb{F}_{2}\right)\right)=\mathbb{F}_{2} \cdot x^{0} .
$$

2. Доказательство теоремы. Компоненты $X_{1}, \ldots, X_{s}$ определяют классы когомологий $\left[X_{1}\right]^{*}, \ldots,\left[X_{s}\right]^{*} \in H^{n}\left(X(\mathbb{C}), \mathbb{F}_{2}\right)$, которые мы будем обозначать через $x_{1}, \ldots, x_{s}$. Необходимо показать, что среди классов когомологий $x_{1}, \ldots, x_{s}$ может быть только одно соотношение $x_{1}+\cdots+x_{s}=0$. Компоненты $X_{1}, \ldots, X_{s}$ определяют также эквивариантные классы когомологий $\left[X_{1}, G\right]^{*}, \ldots,\left[X_{s}, G\right]^{*} \in H^{n}\left(X(\mathbb{C}) ; G, \mathbb{F}_{2}\right)$ (см. [3], [4]), мы будем обозначать их через $\boldsymbol{x}_{1}, \ldots, \boldsymbol{x}_{s}$. При гомоморфизме забывания вещественной структуры

$$
\alpha^{n}: H^{n}\left(X(\mathbb{C}) ; G, \mathbb{F}_{2}\right) \rightarrow H^{n}\left(X(\mathbb{C}), \mathbb{F}_{2}\right)
$$

эквивариантные классы когомологий $\boldsymbol{x}_{1}, \ldots, \boldsymbol{x}_{s}$ переходят в обычные классы когомологий $x_{1}, \ldots, x_{s}$. При гомоморфизме ограничения

$$
\beta^{n}: H^{n}\left(X(\mathbb{C}) ; G, \mathbb{F}_{2}\right) \rightarrow H^{n}\left(X(\mathbb{R}) ; G, \mathbb{F}_{2}\right)=H^{*}\left(X(\mathbb{R}), \mathbb{F}_{2}\right)=\bigoplus_{i=1}^{s} H^{*}\left(X_{i}, \mathbb{F}_{2}\right)
$$

эквивариантньй класс когомологий $\boldsymbol{x}_{i}$ переходит в полньй класс Штифеля-Уитни $w\left(X_{i}\right) \in H^{*}\left(X_{i}, \mathbb{F}_{2}\right)$ (см. [3]). Поэтому выполняется равенство

$$
\beta^{n, 0}\left(\boldsymbol{x}_{i}\right)=x_{i}^{0}
$$


где $x_{i}^{0} \in H^{0}\left(X_{i}, \mathbb{F}_{2}\right)$ - класс когомологий, которьй на каждом одноточечном цикле на $X_{i}$ принимает значение 1. Далее будем рассуждать от противного. Предположим, что сушествует соотношение $x_{i_{1}}+\cdots+x_{i_{r}}=0$, где $r<s$. Тогда

$$
\boldsymbol{x}_{i_{1}}+\cdots+\boldsymbol{x}_{i_{r}} \in K^{n}\left(X(\mathbb{C}) ; G, \mathbb{F}_{2}\right),
$$

а в силу равенства (6) получаем

$$
\beta^{n, 0}\left(\boldsymbol{x}_{i_{1}}+\cdots+\boldsymbol{x}_{i_{r}}\right)=0 \quad \text { или } \quad \beta^{n, 0}\left(\boldsymbol{x}_{i_{1}}+\cdots+\boldsymbol{x}_{i_{r}}\right)=x^{0} .
$$

Однако, из формулы (7) имеем равенство

$$
\beta^{n, 0}\left(\boldsymbol{x}_{i_{1}}+\cdots+\boldsymbol{x}_{i_{r}}\right)=x_{i_{1}}^{0}+\cdots+x_{i_{r}}^{0},
$$

правая часть которого не равна 0. Осталось заметить, что из условия $r<s$ следует неравенство $x_{i_{1}}^{0}+\cdots+x_{i_{r}}^{0} \neq x^{0}$. Теорема доказана.

\section{СПИСОК ЦИТИРОВАННОЙ ЛИТЕРАТУРЫ}

[1] Харламов В. М. Топологические типы неособых поверхностей степени 4 в $\mathbb{R P}^{3} / /$ Функцион. анализ и его прилож. 1976. Т. 10. № 4. С. 55-68.

[2] Краснов В.А. О классах гомологий, определенных вещественньми точками вещественного алгебраического многообразия // Изв. АН СССР. Сер. матем. 1991. Т. 55. №2 2. С. 282-302.

[3] Краснов В. А. Об эквивариантных когомологиях Гротендика вещественного алгебраического многообразия и их приложениях // Изв. РАН. Сер. матем. 1994. Т. 58. № 3. С. 36-52.

[4] Краснов В.А. О классах когомологий, определенных вещественными точками вещественной алгебраической GM-поверхности // Изв. РАН. Сер. матем. 1993. Т. 57. № 5. С. 210-221.

Ярославский государственный университет

Поступило 06.03 .98 